\title{
Is the giant radio galaxy M 87 a TeV gamma-ray emitter?
}

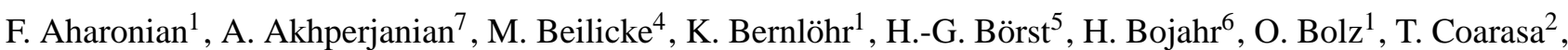
J. L. Contreras ${ }^{3}$, J. Cortina ${ }^{10}$, S. Denninghoff ${ }^{2}$, M. V. Fonseca ${ }^{3}$, M. Girma ${ }^{1}$, N. Götting ${ }^{4}$, G. Heinzelmann ${ }^{4}$, G. Hermann ${ }^{1}$, A. Heusler ${ }^{1}$, W. Hofmann ${ }^{1}$, D. Horns ${ }^{1}$, I. Jung ${ }^{1}$, R. Kankanyan ${ }^{1}$, M. Kestel ${ }^{2}$, A. Kohnle ${ }^{1}$, A. Konopelko ${ }^{1}$, H. Kornmeyer ${ }^{2}$, D. Kranich ${ }^{2}$, H. Lampeitl ${ }^{4}$, M. Lopez ${ }^{3}$, E. Lorenz ${ }^{2}$, F. Lucarelli $^{3}$, O. Mang ${ }^{5}$, H. Meyer ${ }^{6}$, R. Mirzoyan ${ }^{2}$, A. Moralejo ${ }^{3}$, E. Ona-Wilhelmi ${ }^{3}$, M. Panter ${ }^{1}$, A. Plyasheshnikov ${ }^{1,8}$, G. Pühlhofer ${ }^{1}$, R. de los Reyes ${ }^{3}$, W. Rhode ${ }^{6}$, J. Ripken ${ }^{4}$, G. Rowell ${ }^{1}$, V. Sahakian ${ }^{7}$, M. Samorski ${ }^{5}$, M. Schilling ${ }^{5}$, M. Siems ${ }^{5}$, D. Sobzynska ${ }^{2,9}$, W. Stamm ${ }^{5}$, M. Tluczykont ${ }^{4}$, V. Vitale ${ }^{2}$, H. J. Völk ${ }^{1}$, C. A. Wiedner ${ }^{1}$, and W. Wittek ${ }^{2}$

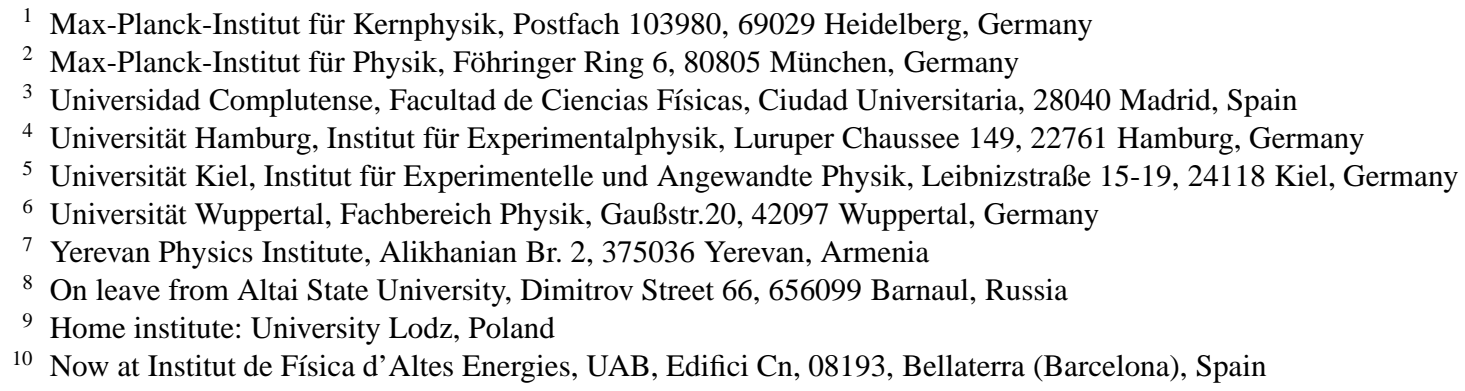

Received 10 February 2003 / Accepted 12 March 2003

\begin{abstract}
For the first time an excess of photons above an energy threshold of $730 \mathrm{GeV}$ from the giant radio galaxy M 87 has been measured at a significance level above $4 \sigma$. The data have been taken during the years 1998 and 1999 with the HEGRA stereoscopic system of 5 imaging atmospheric Cherenkov telescopes. The excess of $107.4 \pm 26.8$ events above $730 \mathrm{GeV}$ corresponds to an integral flux of $3.3 \%$ of the Crab flux or $N_{\gamma}(E>730 \mathrm{GeV})=(0.96 \pm 0.23) \times 10^{-12} \mathrm{phot} \mathrm{cm}^{-2} \mathrm{~s}^{-1}$. M 87 is located at the center of the Virgo cluster of galaxies at a relatively small redshift of $z=0.00436$ and is a promising candidate among the class of giant radio galaxies for the emission of $\mathrm{TeV} \gamma$-radiation. The detection of $\mathrm{TeV} \gamma$-rays from M $87-$ if confirmed - would establish a new class of extragalactic source in this energy regime since all other AGN detected to date at $\mathrm{TeV}$ energies are BL Lac type objects.
\end{abstract}

Key words. $\gamma$-rays: observations - galaxies: individual: M 87

\section{Introduction}

Active Galactic Nuclei (AGN) are believed to contain as a central "engine" a supermassive black hole which causes the development of large scale jets. Extragalactic $\mathrm{TeV} \gamma$-ray emission has been observed so far from AGN only of the BL Lac type, i.e. objects ejecting matter in a jet oriented very close to the observer's line of sight. In BL Lacs, TeV photons are commonly believed to originate in the relativistic jets, most popularly due to inverse Compton scattering. The well studied objects Mkn 421 (redshift $z=0.030$ ) and Mkn 501 $(z=0.034)$ belong to this type of $\mathrm{TeV} \gamma$-ray emitters. Recently, the BL Lac type objects 1ES $1959+650(z=0.047)$ (Nishiyama et al. 1999; Aharonian et al. 2003b) and the much more distant H 1426+428 ( $z=0.129$ ) (Horan et al. 2002; Aharonian et al. 2002a) have also been established as $\mathrm{TeV} \gamma$-ray emitters.

Send offprint requests to: N. Götting, M. Tluczykont e-mail: [Niels.Goetting; Martin.Tluczykont]@desy.de
However, other types of AGN, e.g. giant radio galaxies, also show relativistic mass outflows, though, in contrast to BL Lac type objects, under large viewing angles. Amongst these the nearby radio galaxy M 87 has been speculated to be a powerful accelerator of cosmic rays (including the highest energy particles observed in the universe, see e.g. Ginzburg \& Syrovatskii 1964; Biermann et al. 2000). M 87 has been targeted with the HEGRA Cherenkov telescopes as one of the prime candidates for $\mathrm{TeV} \gamma$-ray emission from this class of objects.

The elliptical galaxy M87 (right ascension $\alpha_{\mathrm{J} 2000.0}$ : $12^{\mathrm{h}} 30^{\mathrm{m}} 49.4^{\mathrm{s}}$, declination $\delta_{\mathrm{J} 2000.0}:+12^{\circ} 23^{\prime} 28^{\prime \prime}$, redshift $z=$ 0.00436 ) has an optical extension of $8.3^{\prime} \times 6.6^{\prime}$ (Ma et al. 1998) with a large radio halo of $16^{\prime} \times 12^{\prime}$ (Cameron 1971). M 87 contains a supermassive black hole with a mass $M_{\mathrm{BH}} \approx 2-3 \times 10^{9} M_{\odot}$ (Harms et al. 1994). The power of the non-thermal jet is estimated to be as high as a few $10^{44} \mathrm{erg} \mathrm{s}^{-1}$ (Owen et al. 2000a). The angle of the M 87 jet axis to the line of sight was determined to be $30^{\circ}-35^{\circ}$ 
Table 1. Dates of individual HEGRA observation periods of M 87. Listed are observation times and mean zenith angles $\langle\vartheta\rangle$. Typically, each night comprises approx. $1-2 \mathrm{~h}$ of observation time.

\begin{tabular}{lcrc}
\hline \hline Date & Year & $\begin{array}{c}\text { Obs. Time } \\
{[\mathrm{h}]}\end{array}$ & $\begin{array}{c}\langle\vartheta\rangle \\
{\left[{ }^{\circ}\right]}\end{array}$ \\
\hline December 28 & 1998 & 0.7 & 23.6 \\
Jan. 17-Jan. 26 & 1999 & 10.7 & 18.4 \\
February 12 & 1999 & 0.7 & 17.0 \\
March 16-March 24 & 1999 & 21.7 & 21.4 \\
April 5-April 21 & 1999 & 29.4 & 23.2 \\
May 8-May 18 & 1999 & 19.9 & 20.9 \\
June 3 & 1999 & 0.3 & 40.5 \\
\hline Total & & 83.4 & 21.6 \\
\hline
\end{tabular}

(Bicknell \& Begelman 1996). M 87 is located in the central region of the Virgo cluster of galaxies, which itself is another interesting site for particle acceleration (e.g. Völk et al. 1996).

The VERITAS collaboration has targeted M 87 with the Whipple $10 \mathrm{~m}$ Cherenkov telescope in the years 2000 and 2001 for a total time of $14 \mathrm{~h}$. Positive excesses have been observed at low significances of $1.6 \sigma$ (2000) and $0.9 \sigma$ (2001) leading to a $3 \sigma$ upper limit of $N_{\gamma}(E>250 \mathrm{GeV})<2.2 \times$ $10^{-11}$ phot cm $\mathrm{cm}^{-2} \mathrm{~s}^{-1}$ (Lebohec et al. 2001).

The HEGRA collaboration has extensively observed M 87 in 1998 and 1999 with the stereoscopic system of 5 imaging atmospheric Cherenkov telescopes (IACT system, Daum et al. 1997). About half of the total observation time ( $44.1 \mathrm{~h}$ out of $83.4 \mathrm{~h}$ ) has been used in an earlier analysis (Götting et al. 2001). No evidence for $\mathrm{TeV}$ emission was found in this dataset and a $3 \sigma$ upper limit on the TeV $\gamma$-ray flux from M 87 was determined to be $N_{\gamma}(E>720 \mathrm{GeV})<1.45 \times 10^{-12}$ phot $\mathrm{cm}^{-2} \mathrm{~s}^{-1}$.

In this Letter the results of the whole data set of the extensive HEGRA M 87 observations during the years 1998 and 1999 are reported, now also applying a more sensitive analysis method. Astrophysical conclusions concerning the nature of the observed excess are briefly discussed.

\section{Observations and results of analysis}

M 87 was observed in the years 1998 and 1999 with the HEGRA IACT system for a total of $83.4 \mathrm{~h}$. There were no further observations of M 87 with the HEGRA telescopes in the subsequent years. The major part of the M 87 data was taken with a 4-telescope setup. Table 1 specifies the observation times and mean zenith angles of the individual HEGRA observation periods. The mean zenith angle of $21.6^{\circ}$ can be converted into a mean energy threshold (defined as the peak detection rate for $\gamma$-showers) of $730 \mathrm{GeV}$ for a Crab-like spectrum (Konopelko et al. 1999).

Only data of good quality were considered for the analysis, the most critical condition being the IACT system's cosmic ray background trigger rate not deviating more than $30 \%$ from the
Table 2. Cuts, event numbers, and significances for the HEGRA observations of M 87 resulting from the signal search using the ring segment and template background model, respectively (see text).

\begin{tabular}{lll}
\hline \hline & M 87 event selection \\
\hline stereo algorithm & $\# 3$ & \\
number of images per event & $\geq 2$ & \\
shape cut on mscw & $<1.1$ & \\
angular distance cut $\Theta^{2}$ & $<0.016 \mathrm{deg}^{2}$ & \\
\hline \multicolumn{3}{l}{ background models: } \\
\cline { 2 - 2 }$N_{\mathrm{ON}}$ & ring segment & template \\
$N_{\mathrm{OFF}}$ & 716 & 716 \\
$\alpha=\Omega_{\mathrm{ON}} / \Omega_{\mathrm{OFF}}$ & 6950 & 1850 \\
$N_{\gamma \text {-candidates }}$ & 0.08757 & 0.325 \\
significance $(\sigma)$ & $107.4 \pm 26.8$ & $114.8 \pm 30.2$ \\
\hline
\end{tabular}

rate expected for the current zenith angle. A total of about 5\% of the data was rejected due to this selection.

All observations of M 87 were carried out in the so-called wobble mode targeting the object's position ("ON") as given in Sect. 1 shifted by $\pm 0.5^{\circ}$ in declination with respect to the center of the field of view. This observation mode allows for simultaneous estimation of the background ("OFF") rate induced by charged cosmic rays (Aharonian et al. 1997). The analysis uses an extended OFF-region reducing the statistical error on the number of background events. A ring segment is chosen with $260^{\circ}$ opening angle at the same radial distance to the center of the field of view as for the $\mathrm{ON}$-source position (see also Aharonian et al. 2002b). The width of the ring is set to the diameter of the $\mathrm{ON}$-source area in order to provide the same angular acceptance for $\mathrm{ON}$ - resp. OFF-source events. This ring segment background model is similar to the usage of a set of control regions (Aharonian et al. 2001), but provides a smaller ratio of ON- to OFF-source solid angle areas $\left(\alpha=\Omega_{\mathrm{ON}} / \Omega_{\mathrm{OFF}}\right)$ and thus reduces the statistical error on the number of estimated background events. For a consistency check (and for a search for $\gamma$-ray sources in the field of view, see below) the so-called template background model has also been used (Rowell 2003, see also Aharonian et al. 2002c).

For the image analysis, the mirror reflectivities and photocathode efficiencies - which degrade slowly with time along with the factors converting from digitized photomultiplier signals to photoelectrons have been determined on a monthly basis. The shower reconstruction and the event selection cuts have already been described in previous publications (e.g. Aharonian et al. 1999). The stereo air shower direction reconstruction algorithm \#3 (Hofmann et al. 1999) and a "tight shape cut" (parameter mscw < 1.1) (Konopelko et al. 1999) for an effective $\gamma$-hadron separation have been applied leading to a sensitivity gain as compared to the earlier analysis of the HEGRA M 87 observations. The optimum angular cut was derived using $\gamma$-ray events from the Crab nebula on the basis of a nearly contemporaneous data set at similar zenith angles. 


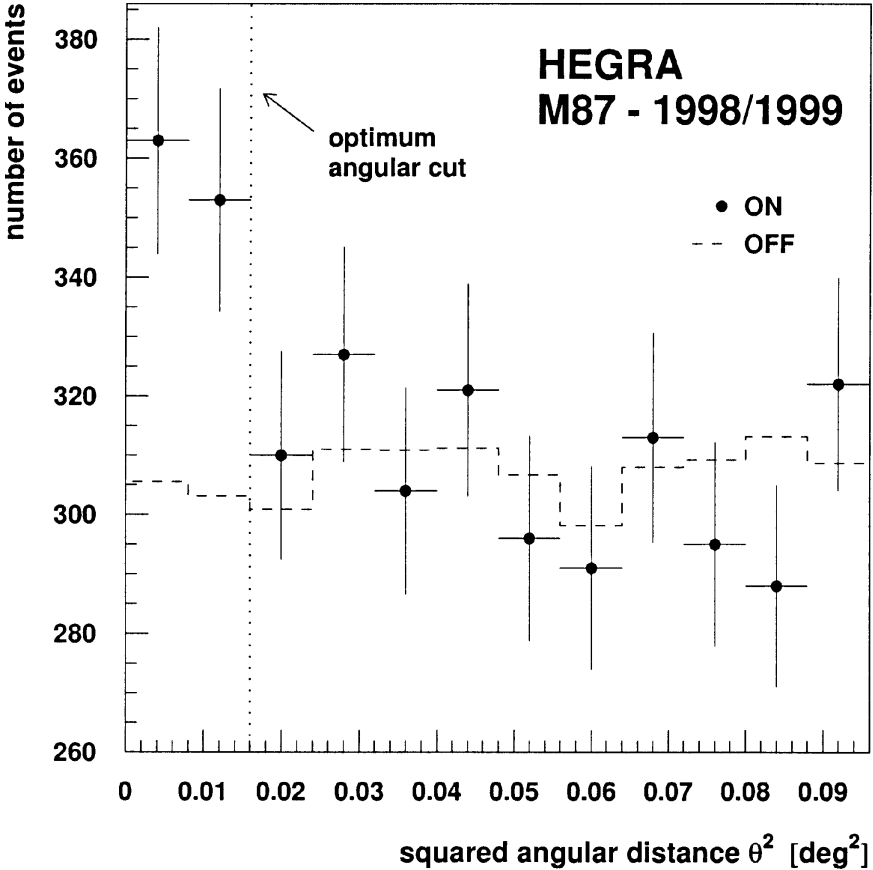

Fig. 1. Number of events vs. squared angular distance $\Theta^{2}$ to the position of M 87 as observed in the years 1998 and 1999 with the HEGRA IACT system. The dots show the $\mathrm{ON}$-source events, while the dashed histogram gives the background estimate determined from a ring segment as explained in the text. The statistical error for the background estimate is much smaller than the error of the $\mathrm{ON}$-source distribution. Indicated by the vertical dotted line is the optimum angular cut as determined from nearly contemporaneous Crab observations at similar zenith angles. The significance of the M 87 excess amounts to $4.1 \sigma$.

Table 2 summarizes the event selection cuts, the resulting event numbers and significances for the data set as derived using the ring segment and template background models.

Figure 1 shows the event distribution both for the $\mathrm{ON}$-source and the OFF-source regions as a function of the squared angular distance of the reconstructed shower direction to the source position after applying all event selection cuts. The statistical significance of the observed excess from the direction of M 87 (at the reference coordinates given in Sect. 1) is $4.1 \sigma$, calculated using formula (17) from $\mathrm{Li} \& \mathrm{Ma}(1983)$. On the basis of the limited event statistics the excess is compatible with a point-like source for the HEGRA IACT system at the position of M 87, although extended emission cannot be excluded. After background subtraction the $m s c w$ values show a Gaussian distribution around the value of 1.0 as expected for a $\gamma$-ray population (see Konopelko et al. 1999). This test supports the hypothesis that the measured excess is a result of M 87 being a true $\mathrm{TeV} \gamma$-ray source. Applying different statistical tests in order to search for burstlike behaviour of M 87 no evidence for flux variations in the $\mathrm{TeV}$ energy range has been found.

The event distribution in the field of view was used to determine the center of gravity position $(\mathrm{CoG})$ of the $\mathrm{TeV} \gamma$ ray excess at $\alpha_{\mathrm{J} 2000.0}=12^{\mathrm{h}} 30^{\mathrm{m}} 54.4^{\mathrm{s}} \pm 6.9_{\text {stat }}^{\mathrm{s}} \pm 1.7_{\text {syst }}^{\mathrm{s}}, \delta_{\mathrm{J} 2000.0}=$ $12^{\circ} 24^{\prime} 17^{\prime \prime} \pm 1.7_{\text {stat }}^{\prime} \pm 0.4_{\text {syst }}^{\prime}$ as shown in Fig. 2 . The accuracy of the CoG determination is limited by a systematic pointing error of about 25" (Pühlhofer et al. 1997). Within the large errors,

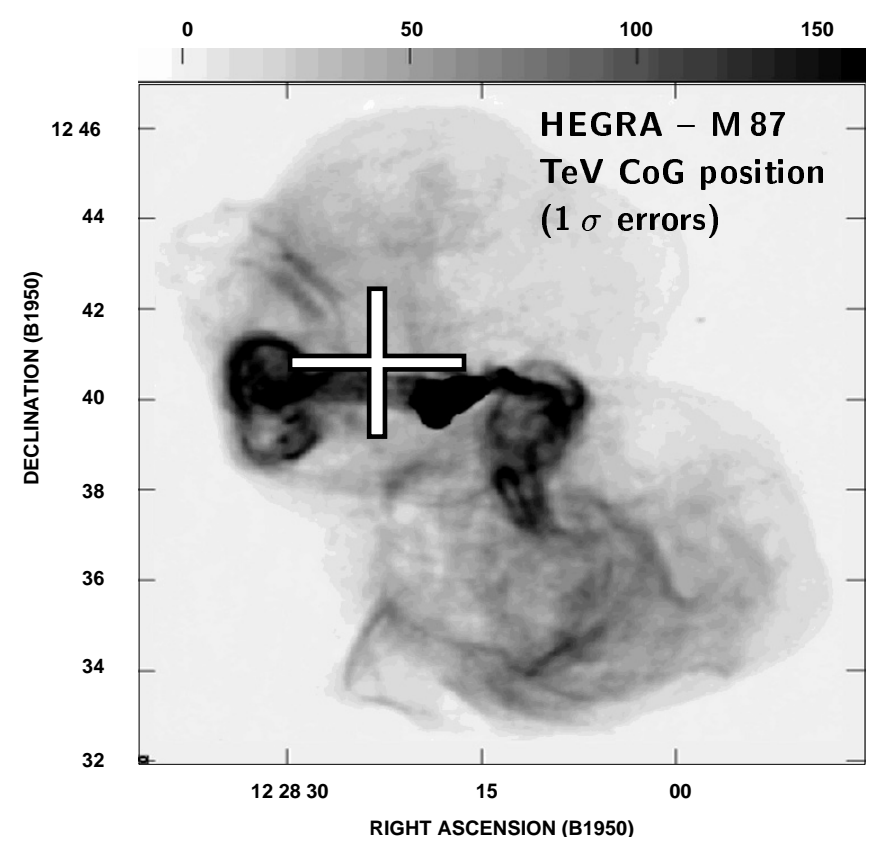

Fig. 2. Radio image of M 87 at $90 \mathrm{~cm}$ showing the structure of the M 87 halo. The center of gravity position of the $\mathrm{TeV} \gamma$-ray excess from the HEGRA M 87 observations is marked by the cross indicating the statistical $1 \sigma$ errors (radio image adapted from Owen et al. 2000b).

the CoG is consistent with the M 87 position, although a small shift of the source position cannot be ruled out. Therefore, it is not possible to localize a candidate $\mathrm{TeV} \gamma$-ray production site to particlular inner radio structures of M 87.

In order to search for $\mathrm{TeV} \gamma$-ray sources in the relatively large field of view of the HEGRA IACT system (and for a consistency check with the ring segment background model), the template background model was used for a further analysis of the M 87 data. A $2^{\circ} \times 2^{\circ}$ skymap of excess events determined for this sky region is shown in Fig. 3. The excess from the direction of M 87 is clearly visible in the representation using overlapping circular bins, showing that the only significant excess in the field of view is related to M 87. The significance using the template model is $3.9 \sigma$ as given in Table 2 . The two background models applied in this analysis use widely different approaches, supporting the assumption that the M 87 excess does not stem from a background fluctuation.

The observed excess can be converted into an integral flux of $(3.3 \pm 0.8) \%$ of the Crab nebula flux (only the statistical error is given because of the low statistics). A conversion into absolute flux units using the well measured photon flux and spectrum of the Crab nebula around $1 \mathrm{TeV}$ (e.g. Aharonian et al. 2000) results in a $\gamma$-ray flux of

$$
N_{\gamma}(E>730 \mathrm{GeV})=(0.96 \pm 0.23) \times 10^{-12} \mathrm{phot} \mathrm{cm}^{-2} \mathrm{~s}^{-1}
$$

A spectral analysis of the data of the M 87 data has been performed using the analysis technique described in Aharonian et al. (2003a). The data can be well described with a power law $\mathrm{d} N / \mathrm{d} E \sim E^{-\alpha}$ with $\alpha=2.9 \pm 0.8_{\text {stat }} \pm 0.08_{\text {syst }}$. The large statistical error results from the low event statistics. 


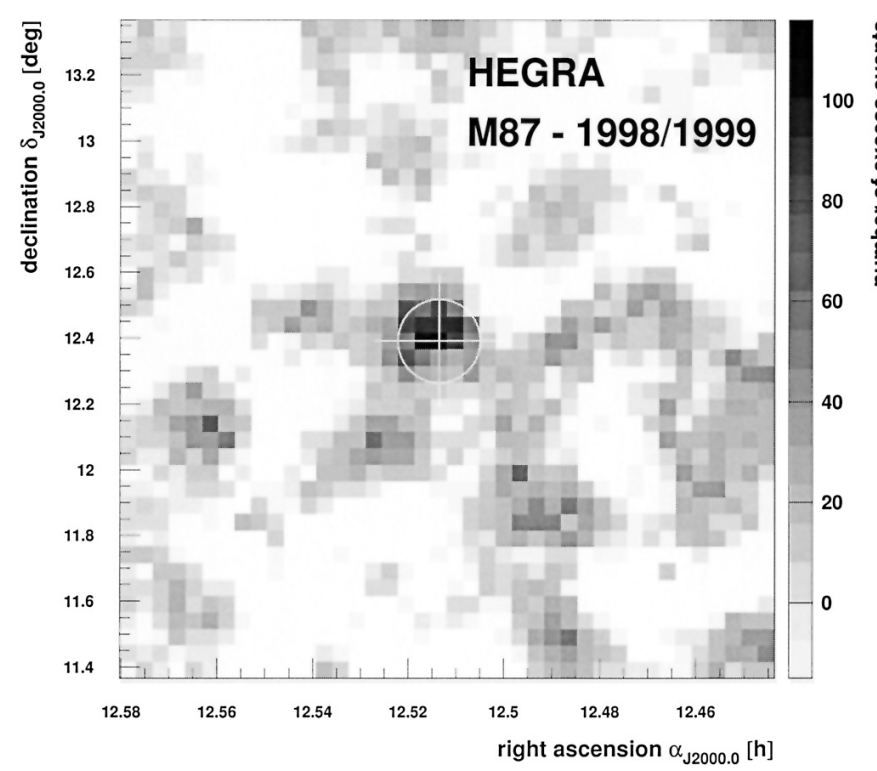

Fig. 3. Skymap $\left(2^{\circ} \times 2^{\circ}\right.$ view at a $0.02^{\circ} \times 0.02^{\circ}$ binning $)$ of the number of excess events in the HEGRA field of view around the position of M 87 generated using the template background model. At each bin, the excess is determined from events within the optimum radius of $0.127^{\circ}$ (based on Crab observations). Negative excesses below -15 events are shown in white color for clarity reasons. The solid angle resulting from the optimum radius is indicated by the white circle with $\mathrm{M} 87$ being located at the intersection of the white lines.

\section{Summary and conclusions}

The radio galaxy M 87 has been observed with the HEGRA IACT system for a total of $83.4 \mathrm{~h}$. For the first time a significant excess of $4.1 \sigma$ has been detected at energies above a mean energy threshold of $730 \mathrm{GeV}$ from a member of this class of objects using the imaging atmospheric Cherenkov technique. Note that nearly 30 years ago the radio galaxy Centaurus A was reported to be a TeV source observed with a non-imaging instrument (Grindlay et al. 1975).

Due to the limited number of excess events detected by the HEGRA telescopes, an analysis of the spectral shape results in a very large statistical error, thus making it difficult to draw a conclusion about the origin of the $\mathrm{TeV} \gamma$-radiation. Assuming a power-law shape with a photon spectral index of 2.9 the integral photon flux at the level of $(3.3 \pm 0.8) \%$ of the Crab nebula flux converts into an energy flux of

$$
F_{\gamma}(E>730 \mathrm{GeV})=(4.3 \pm 1.0) \times 10^{-12} \mathrm{erg} \mathrm{cm}^{-2} \mathrm{~s}^{-1} .
$$

Given the distance to M 87 of about $16 \mathrm{Mpc}$, this corresponds to a $\gamma$-ray luminosity above $730 \mathrm{GeV}$ of about $10^{41} \mathrm{erg} \mathrm{s}^{-1}$ under the assumption of isotropic emission.

Several different possibilites for the origin of $\mathrm{GeV} / \mathrm{TeV}$ $\gamma$-radiation are conceivable. M 87 with its pc scale jet has recently been modeled within the Synchrotron Self Compton scenario as a BL Lac object seen at a large angle to its jet axis (Bai \& Lee 2001). It has also been modeled using the so-called Synchrotron Proton Blazar model (Protheroe et al. 2002). In both models, the observed flux can be accommodated.

The large scale $(\mathrm{kpc})$ jets with several knots detected at radio to X-ray frequencies and believed to have synchrotron origin due to electrons with energies up to $100 \mathrm{TeV}$ is also a possible $\gamma$-ray production site in M 87. Consequently, inverse Compton $\gamma$-rays in the $1-10 \mathrm{TeV}$ energy range can be expected within reasonable model parameters.

Moreover, $\gamma$-rays could be produced in the interstellar medium of M 87, i.e. at larger distance scales from the center of this active galaxy. Both inverse Compton and hadronic interactions could generate a $\mathrm{TeV} \gamma$-ray luminosity in the observed range of approx. $10^{41} \mathrm{erg} \mathrm{s}^{-1}$. If this interpretation is valid, one would expect a slightly extended source $\left(2-3^{\prime}\right)$.

It should be noted that M 87 is also considered as a possible source of $\mathrm{TeV} \gamma$-rays from the hypothetical neutralino annihilation process (Baltz et al. 2000).

A weak signal at the centi-Crab level is at the sensitivity threshold for the HEGRA IACT system for observation times of the order of $100 \mathrm{~h}$. Therefore, a deep investigation and possible confirmation with a spectral analysis of the M 87 excess should be subject of the next generation Cherenkov telescope projects like H.E.S.S, MAGIC and VERITAS, which provide increased sensitivity together with a lower energy threshold. Due to the proximity to M $87(16 \mathrm{Mpc})$ and to the increased accuracy of these observations (a fraction of an arc minute), these measurements may allow the location of the $\gamma$-ray production site in M 87 to be more accurately determined thus greatly advancing our understanding of its $\mathrm{TeV} \gamma$-radiation.

Acknowledgements. The support of the German Federal Ministry for Research and Technology BMBF and of the Spanish Research Council CICYT is gratefully acknowledged. G. Rowell acknowledges receipt of a von Humboldt fellowship. We thank the Instituto de Astrofísica de Canarias (IAC) for the use of the HEGRA site at the Observatorio del Roque de los Muchachos (ORM) and for supplying excellent working conditions on La Palma.

\section{References}

Aharonian, F. A., Akhperjanian, A. G., Barrio, J. A., et al. 1997, A\&A, 327, L5

Aharonian, F. A., Akhperjanian, A. G., Barrio, J. A., et al. 1999, A\&A, 342, 69

Aharonian, F. A., Akhperjanian, A. G., Barrio, J. A., et al. 2000, ApJ, 539,317

Aharonian, F. A., Akhperjanian, A. G., Barrio, J. A., et al. 2001, A\&A, 370,112

Aharonian, F. A., Akhperjanian, A. G., Barrio, J. A., et al. 2002a, A\&A, 384, L23

Aharonian, F. A., Akhperjanian, A. G., Beilicke, M., et al. 2002b, A\&A, 393, 89

Aharonian, F. A., Akhperjanian, A. G., Beilicke, M., et al. 2002c, A\&A, 393, L37

Aharonian, F. A., Akhperjanian, A. G., Beilicke, M., et al. 2003a, A\&A, submitted [astro-ph/0301437]

Aharonian, F. A., Akhperjanian, A. G., Beilicke, M., et al. 2003b, in preparation

Bai, J. M., \& Lee, M. G. 2001, ApJ, 549, L173

Baltz, E. A., Briot, C., Salati, P., et al. 2000, Phys. Rev. D, 61, 023514

Bicknell, G. V., \& Begelman, M. C. 1996, ApJ, 467, 597

Biermann, P. L., Ahn, E.-J., Medina-Tanco, G., et al. 2000, Nucl. Phys. B, Proc. Suppl., 87, 417 
Cameron, M. J. 1971, MNRAS, 152, 439

Daum, A., Hermann, G., Heß, M., et al. 1997, Astropart. Phys., 8, 1

Ginzburg, V. L., \& Syrovatskii, S. I. 1964, The Origin of Cosmic Rays (New York: Pergamon Press)

Götting, N., Robrade, J., \& the HEGRA collaboration 2001, Proc. of the 27th ICRC, Hamburg 2001, OG 2.3.199, 7, 2669

Grindlay, J. E., Helmken, H. F., Brown, R. H., et al. 1975, ApJ, 197, L9

Harms, R. J., Ford, H. C., Tsvetanov, Z. I., et al. 1994, ApJ, 435, L35

Hofmann, W., Jung, I., Konopelko, A., et al. 1999, Astropart. Physics, 12, 135

Horan, D., Badran, H. M., Bond, I. H., et al. 2002, ApJ, 571, 753

Konopelko, A., Hemberger, M., Aharonian, F. A., et al. 1999, Astropart. Physics, 10, $275 \mathrm{ff}$.

Lebohec, S., et al. 2001, Proc. of the 27th ICRC, Hamburg 2001, OG 2.3.191, 7, 2643
Li, T.-P., \& Ma, Y.-Q. 1983, ApJ, 272, 317

Ma, C., Arias, E. F., Eubanks, T. M., et al. 1998, AJ, 116, 516

Nishiyama, T., Chamoto, N., Chiwaka, M., et al. 1999, in Proc. of the 26th ICRC, Salt Lake City, 3, 370

Owen, F. N., Eilek, J. A., \& Kassim, N. E., et al. 2000a, ApJ, 543, 611

Owen, F. N., Ledlow, M. J., Eilek, J. A., et al. 2000b, Proc. of The Universe at Low Radio Frequencies, ASP Conf. Ser., 199, also [astro-ph/0006152]

Pühlhofer, G., Daum, A., Hermann, G., et al. 1997, Astropart. Phys., 8,101

Protheroe, R. J., Donea, A.-C., \& Reimer, A. 2002, Astropart. Phys., accepted [astro-ph/0210249]

Rowell, G. 2003, in preparation

Völk, H. J., Aharonian, F. A., \& Breitschwerdt, D. 1996, Space Sci. Rev., 75, 279 